\title{
Factor B structure provides insights into activation of the central protease of the complement system

\author{
Fin J Milder ${ }^{1}$, Lucio Gomes ${ }^{1}$, Arie Schouten ${ }^{1}$, Bert J C Janssen ${ }^{1}$, Eric G Huizinga ${ }^{1}$, Roland A Romijn ${ }^{2}$, \\ Wieger Hemrika ${ }^{2}$, Anja Roos ${ }^{3}$, Mohamed R Daha ${ }^{3}$ \& Piet Gros ${ }^{1}$
} \\ Factor $B$ is the central protease of the complement system of immune defense. Here, we present the crystal structure of human factor $B$ at 2.3- $\AA$ resolution, which reveals how the five-domain proenzyme is kept securely inactive. The canonical activation helix of the Von Willebrand factor A (VWA) domain is displaced by a helix from the preceding domain linker. The two helices conformationally link the scissile-activation peptide and the metal ion-dependent adhesion site required for binding of the ligand $\mathrm{C} 3 \mathrm{~b}$. The data suggest that $\mathrm{C} 3 \mathrm{~b}$ binding displaces the three $\mathrm{N}$-terminal control domains and reshuffles the two central helices. Reshuffling of the helices releases the scissile bond for final proteolytic activation and generates a new interface between the VWA domain and the serine protease domain. This allosteric mechanism is crucial for tight regulation of the complement- amplification step in the immune response.
}

\begin{abstract}
Factor B is a tightly regulated, highly specific serine protease. In its activated form, it catalyzes the central amplification step of complement activation to initiate inflammatory responses, cell lysis, phagocytosis and B-cell stimulation ${ }^{1,2}$. Factor B is activated through an assembly process: it binds surface-bound $\mathrm{C} 3 \mathrm{~b}$, or its fluid-phase counterpart $\mathrm{C} 3\left(\mathrm{H}_{2} \mathrm{O}\right)$, after which it is cleaved by factor $\mathrm{D}$ into fragments $\mathrm{Ba}$ (residues 1-234) and $\mathrm{Bb}$ (residues 235-739) ${ }^{3,4}$. Fragment $\mathrm{Ba}$ dissociates from the complex, leaving behind the alternative pathway $\mathrm{C} 3$ convertase complex $\mathrm{C} 3 \mathrm{~b}-\mathrm{Bb}$, which cleaves $\mathrm{C} 3$ into $\mathrm{C} 3 \mathrm{a}$ and C3b (see Fig. 1a). This protease complex is intrinsically instable. Once dissociated from the complex, $\mathrm{Bb}$ cannot reassociate with $\mathrm{C}_{3} \mathrm{~b}^{5}$. A similar C3 convertase complex is formed upon activation of the classical (antibody-mediated) and lectin-binding pathways, comprised of $\mathrm{C} 4$ and $\mathrm{C} 2$, which are homologous to $\mathrm{C} 3$ and factor $\mathrm{B}$, respectively. The proenzyme factor $\mathrm{B}$ consists of three $\mathrm{N}$-terminal complement control protein (CCP) domains, connected by a 45-residue linker to a VWA domain and a C-terminal serine protease (SP) domain, which carries the catalytic center (Fig. 1a). The VWA and SP domains form fragment $\mathrm{Bb}$, and CCP1 through CCP3 and the linker form fragment $\mathrm{Ba}$. Binding of factor $\mathrm{B}$ to $\mathrm{C} 3 \mathrm{~b}$ depends on elements in fragment $\mathrm{Ba}^{6}$ and the $\mathrm{Mg}^{2+}$-dependent metal ion-dependent adhesion site (MIDAS) motif in the VWA domain of fragment $\mathrm{Bb}^{7}$. The VWA domain is structurally homologous to inserted (I) domains in integrins. In I domains, ligand binding to the MIDAS is coupled to a $\sim 10$ - $\AA$ shift of the $\alpha 7$ activation helix, with concomitant domain rearrangements that activate the integrins ${ }^{8,9}$. Structures of a truncated $\mathrm{Bb}$ fragment ${ }^{10}$ and its full-length homolog $\mathrm{C} 2 \mathrm{a}^{11}$ show variable positions of the $\alpha 7$ activation helix affecting
\end{abstract}

the orientation of the VWA and SP domains, which indicates that a related mechanism may occur in convertase formation and dissociation. These structures, however, do not reveal the regulation of the proteolytic activity of factor B. In particular, it is unclear how factor B is maintained in its inactive, zymogen state and how C3b binding makes factor B susceptible to proteolytic activation by factor D. To gain insights into the regulatory mechanisms underlying complement activation, we set out to determine the structure of human proenzyme factor $\mathrm{B}$.

\section{RESULTS \\ Overall structure of factor B}

Here we present the crystal structure of human proenzyme factor B at 2.3- $\AA$ resolution (see Methods and Supplementary Fig. 1 online). The overall structure (Fig. 1b) consists of three lobes, which is in agreement with electron micrographs ${ }^{12}$. One lobe represents the trypsinlike SP domain with the catalytic site in a fully exposed position. The other two lobes represent the VWA domain, with its $\alpha / \beta / \alpha$ fold, and the CCP1-CCP3 domains, with their typical $\beta$-sandwich fold stabilized by pairs of disulphide bonds. The long CCP3-VWA linker, containing the Arg234-Lys235 scissile bond, forms a short loop (residues 198-201), an $\alpha$-helix (residues 202-213) and a long loop (residues 214-242), which is partially disordered (residues 219-232 are not evident in the electron density). Unexpectedly, the observed linker helix, denoted $\alpha \mathrm{L}$, is integrated into the VWA domain and occupies the position of the $\alpha 7$ activation helix in fragment $\mathrm{Bb}^{10}$ (Fig. 2a). The displaced $\alpha 7$ helix lies adjacent to $\alpha \mathrm{L}$ on the surface of the VWA domain. These two helices lie at the center of the five-domain 
a

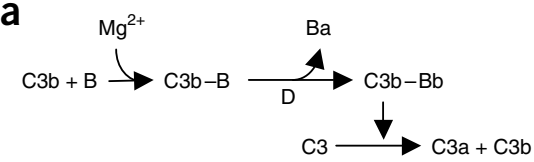

B
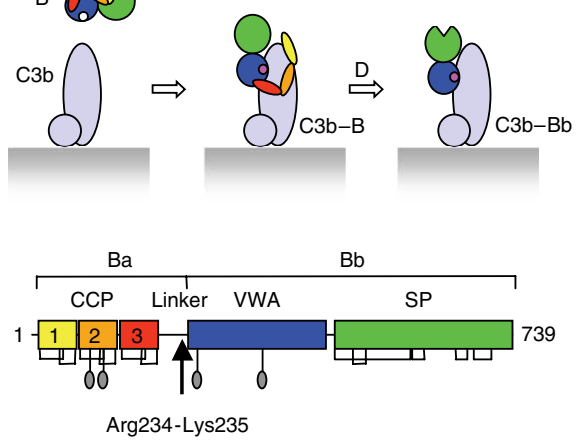

b
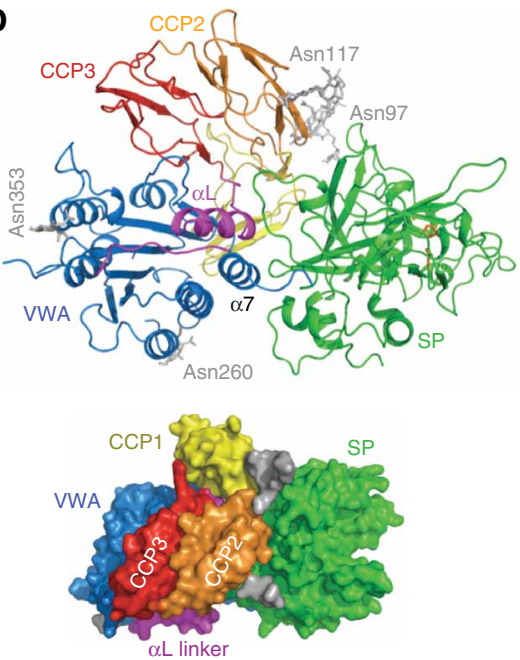

Figure 1 C3 convertase formation and crystal structure of complement factor B. (a) Top and middle diagrams, schematic and cartoon representation (based on crystal structure ${ }^{33,34}$ ) of C3 convertase formation. Bottom diagram, domain topology of factor $\mathrm{B}$; indicated are the $\mathrm{N}$-linked glycans, the disulphide bridges and the Arg234-Lys235 scissile bond. (b) Top, structure of factor $B$ shown as ribbons, colored by domain as in a. The linker connecting CCP3 and VWA is colored purple. The $\mathrm{N}$-linked glycans (gray) and the catalytic triad Asp-His-Ser (brown) are shown as sticks. Labels indicate the centrally positioned helices $\alpha \mathrm{L}$ and $\alpha 7$. Bottom, factor B shown in surface representation (rotated $90^{\circ}$ with respect to the top structure).

dominantly by the conformations of loop $\beta \mathrm{F}-\alpha 7$, helix $\alpha 7$ and the neighboring helix $\alpha 1$ (Fig. 2b $)^{8,13}$. In factor B, helix $\alpha 7$ is displaced from its normal binding groove by

molecule and affect the domain-domain arrangement (Fig. 1b). They provide a conformational link between two key regulatory sites of the molecule: the $\mathrm{Mg}^{2+}$-dependent C3b-binding MIDAS on one side and the activation scissile bond Arg234-Lys235 on the opposite side of the molecule.

\section{VWA domain adopts locked conformation}

The VWA domain in factor B adopts an inactive conformation with a displaced helix $\alpha 7$ and distorted MIDAS, which we refer to as a 'locked' conformation. The inactive-closed and active-open conformational states of the VWA and I domains are characterized pre- helix $\alpha \mathrm{L}$ (Fig. 2a). The preceding loop, $\beta \mathrm{F}-\alpha 7$, adopts a canonical closed conformation, as shown by the positions of its hydrophobic ratchet residues (Val430 and Leu436; see Fig. 2b and Supplementary Fig. 2 online). Helix $\alpha 1$ is also positioned in the inactive-closed conformation. Thus, the unliganded VWA domain has an inactive conformation, as would be expected for the proenzyme state of factor B. Moreover, helix $\alpha \mathrm{L}$ obstructs the $\alpha 7$ activation helix, preventing it from taking its normal position in VWA and I domains $8,10,11,13-15$, thereby locking the VWA domain in its inactive conformation. Typically, a closed-inactive domain conformation is coupled to a MIDAS conformation with a low affinity for ligands. In factor B,
Figure 2 Regulatory elements in the VWA domain. (a) Dislocation of helix $\alpha 7$ by helix $\alpha \mathrm{L}$. Left, overlay of the VWA domains of factor $B$ and fragment $\mathrm{Bb}$ (PDB 1RRK) shows the displacement of helix $\alpha 7$ (blue in factor $B$, green in $\mathrm{Bb}$ ) by linker helix $\alpha \mathrm{L}$ (purple). Right, stereo figure shows the packing of helices $\alpha 7$ and $\alpha \mathrm{L}$ against the body of the VWA domain of factor B (gray, surface representation). Hydrophobic residues of helices $\alpha \mathrm{L}(\mathrm{B})$ and $\alpha 7$ (Bb) are shown in ball-and-stick representation. (b) Conformations of loop $\beta \mathrm{F}-\alpha 7$ and helices $\alpha 7$ and $\alpha 1$. Left image, overlay of VWA domains of factor B (blue) with closed (green; PDB 1AOX) and open (yellow; PDB 1DZI) conformations of the $\alpha_{2}$ I domain, showing strand $\beta F$ and helix $\alpha 7$. Middle two images, positions of hydrophobic 'ratchet' residues (sticks) of loop $\beta \mathrm{F}-\alpha 7$ and helix $\alpha 7$. Right image, position of helix $\alpha 1$. (c) Distortion of the $\mathrm{Mg}^{2+}$-dependent MIDAS C3b binding site. Left, superposition of the MIDAS motif in factor B (blue) and in Bb (gray), shown in ball-and-stick representation. Gray spheres represent water molecules (small spheres) and the bound ion (large sphere) as observed in $\mathrm{Bb}$. Right, orientation of CCP1 (yellow) and VWA (blue) in factor $\mathrm{B}$, with the VWA domain of $\mathrm{Bb}$ (gray) superposed. Shown in ball-and-stick representation are the adjoining residues Gln28 and Asp254 and the glycosylation site Asn260. (d) The $\mathrm{P} 1$ residue of the buried scissile bond (Arg234-Lys235) interacts with helices $\alpha 7$ (blue) and linker helix $\alpha \mathrm{L}$ (purple) via salt bridges.
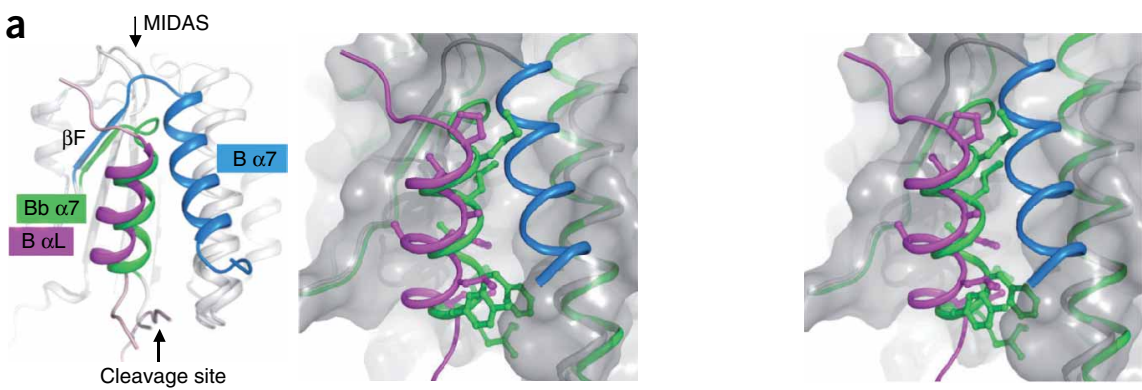

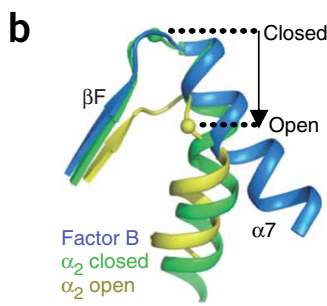

C

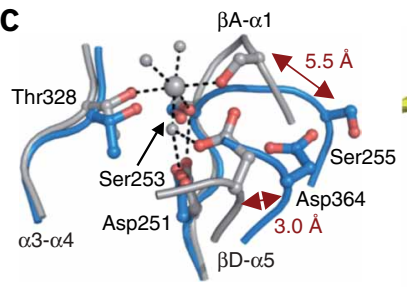

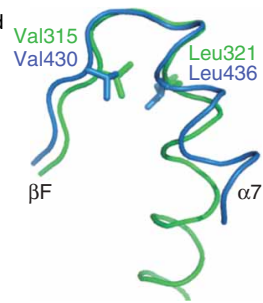

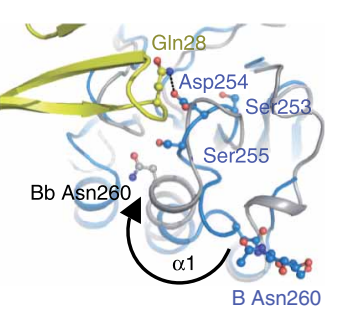

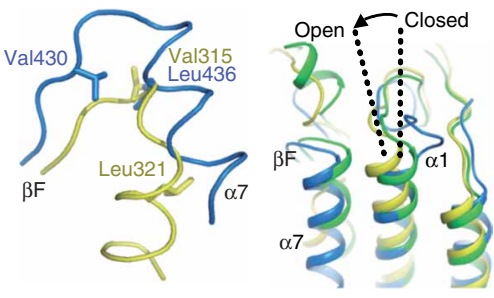

d

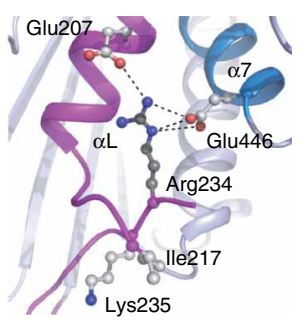




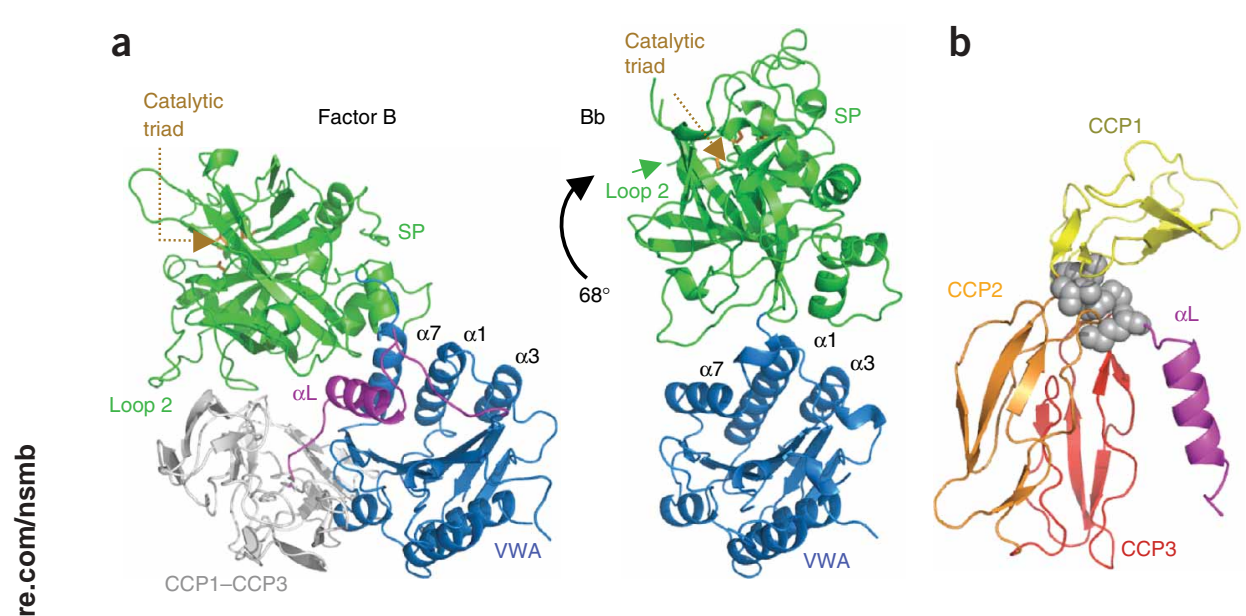

Figure 3 Domain orientations in factor B and Bb. (a) Left, orientation of VWA (blue) and SP (green) in factor $B$ with linker and linker helix $\alpha \mathrm{L}$ shown in purple and CCP1-CCP3 domains in gray. Right, orientation of VWA and SP in Bb (PDB 1RRK). Indicated are helices $\alpha \mathrm{L}, \alpha 1, \alpha 3$ and $\alpha 7$, which are involved in the VWA-SP interfaces. (b) The CCP triad arrangement in ribbon representation, with CCP1 in yellow, CCP2 in orange and CCP3 in red. Gray spheres indicate the four residues (Tyr42, Ile76, Phe197 and Met198) forming the hydrophobic triad center. The adjoining linker containing helix $\alpha \mathrm{L}$ is colored purple.

compared with fragment $\mathrm{Bb}$, displacements up to $5.5 \AA$ in loops $\beta \mathrm{A}$ $\alpha 1$ and $\beta D-\alpha 5$ distort the MIDAS (Fig. $2 c$ and Supplementary Fig. 2). The distorted conformation indicates reduced divalent-ion binding. Moreover, we did not observe electron density for an ion bound to the MIDAS in factor B. The absence of an ion is consistent with the notion that fragment $\mathrm{Bb}$, but not factor $\mathrm{B}$, has high affinity for divalent ions ${ }^{16}$. The neighboring CCP1 domain may affect the MIDAS conformation. Gln 28 of loop $\beta 2-\beta 3$ in CCP1 makes a hydrogen bond to Asp254, located in the displaced VWA $\beta A-\alpha 1$ loop. Furthermore, CCP1 may prevent loop $\beta \mathrm{A}-\alpha 1$ from assuming the conformation seen in $\mathrm{Bb}$ by obstructing the glycan at Asn260. Instead, in factor B helix $\alpha 1$ is shifted and partially unwound, pointing the glycan on Asn260 away from the neighboring CCP1 domain (Fig. 2c). Factor B with D254G and N260D single and double mutations (where N260D deletes the $\mathrm{N}$-glycosylation site) forms convertases more readily and more stably than wild-type factor $\mathrm{B}^{17,18}$, indicating that these mutations promote the transition from an inactive to an active conformation of the VWA domain.

\section{Central role for linker region}

The CCP3-VWA linker helix $\alpha \mathrm{L}$ occupies the position of helix $\alpha 7$ of the VWA domain. It binds Arg234 of the scissile bond, preventing proteolytic activation. By virtue of its short-chain hydrophobic residues, helix $\alpha \mathrm{L}$ is squeezed between helix $\alpha 7$ and the VWA core, effectively blocking helix $\alpha 7$ (Fig. 2a). After helix $\alpha$ L, the CCP3VWA linker forms a long loop and folds back to the C-terminal end of helix $\alpha \mathrm{L}$ (Fig. 2d). The scissile bond Arg234-Lys235 is located here, buried by the preceding flexible loop. The side chain of Arg234, crucial for substrate binding at the S1 pocket (as defined in Schechter and Berger notation) of factor D, is bound to both helix $\alpha \mathrm{L}$ (Glu207) and helix $\alpha 7$ (Glu446) by salt bridges (Fig. 2d). Thus, the scissile bond in factor B is protected from proteolysis by factor D and is conformationally linked to helices $\alpha \mathrm{L}$ and $\alpha 7$. In contrast, the scissile bond in isolated VWA domains is susceptible to factor D cleavage ${ }^{19}$, because they lack the $\alpha \mathrm{L}-\alpha 7$ arrangement that locks the P1 residue (Arg234). In the formation of the convertase, binding of C3b to factor
B probably induces reshuffling of helices $\alpha \mathrm{L}$ and $\alpha 7$ that liberates the $\mathrm{P} 1$ arginine residue and makes the scissile bond accessible for proteolysis by factor $\mathrm{D}$.

Conformation of the catalytic SP domain In both factor $\mathrm{B}$ and fragment $\mathrm{Bb}^{10}$, the $\mathrm{SP}$ domain is exposed and the catalytic site is freely accessible to small substrates ${ }^{20}$. The two structures differ markedly in the orientation of the VWA-SP region (Fig. 3a). In factor B, the VWA-SP interface is dominated by helices $\alpha \mathrm{L}$ and $\alpha 7$, with the $\mathrm{N}$-terminal loop of SP folding over helix $\alpha \mathrm{L}$; furthermore, contacts are observed between SP and domains CCP1 and CCP2. In fragment $\mathrm{Bb}$, where $\alpha \mathrm{L}$ and the CCP1-CCP3 domains are absent, SP rotates $68^{\circ}$ and contacts helices $\alpha 7, \alpha 1$ and $\alpha 3$ of the VWA domain. In addition to the interface loops, loop 2 of the SP domain is markedly different in factor B and fragment Bb. Putatively, Arg705 of this loop (like Arg696 in $\mathrm{C}^{2} \mathrm{a}^{11}$ ) induces formation of the oxyanion hole, similar to the $\mathrm{N}$ terminus in trypsin after proteolytic activation of trypsinogen ${ }^{21}$. Consistent with this model, the guanidinium group of the arginine interacts with an aspartate of the oxyanion-hole loop in $\mathrm{Bb}$ and $\mathrm{C} 2 \mathrm{a}$ (Supplementary Fig. 3 online). In factor B, loop 2 folds back toward the N-glycosylated Asn97 on CCP2 (weak electron density for the side chains indicates disorder in loop 2). Concomitantly, the guanidinium group of Arg705 is displaced by $11 \AA$, as would be expected for the zymogen state. Nevertheless, the conformation of the oxyanion hole is identical between factor $\mathrm{B}$ and fragment $\mathrm{Bb}$ - that is, in factor $\mathrm{B}$, the oxyanion-hole loop does not adopt the zymogen state. This indicates that proteolytic activity of factor B is probably not controlled directly, as in trypsinogens, but rather through quaternary rearrangement of the domains in the assembly process.

\section{Triad of CCP domains}

The three CCP domains of fragment Ba adopt an unexpected triad arrangement, with domains CCP2 and CCP3 packed tightly (Supplementary Table 1 online) into an antiparallel dimer capped by CCP1. This arrangement produces a small hydrophobic core at the triad center (Fig. 3b). Changes in the CCP2-CCP3 interface reduce C3b binding and hemolytic activity ${ }^{22,23}$, and antibodies directed against CCP1 and CCP3 block hemolytic activity ${ }^{22,24}$ (Supplementary Fig. 4 online), indicating that the triad of CCP domains provides an important binding site for $\mathrm{C} 3 \mathrm{~b}$. Domain CCP1 probably hinders access of the ligand $\mathrm{C} 3 \mathrm{~b}$ to the $\mathrm{Mg}^{2+}$-dependent MIDAS of the VWA domain (Supplementary Fig. 5 online). Because the triad of CCP domains appears to be only weakly associated with the VWA and SP domains (Supplementary Fig. 4 and Supplementary Table 2 online), we hypothesize that binding of the CCP domains to C3b dislocates the CCP triad from the VWA and SP domains. Concomitantly, dislocation of the CCP triad may be coupled, through the short CCP3- $\alpha$ L connecting loop (Fig. 3b), to displacement of helix $\alpha$ L from its binding groove in the VWA domain.

\section{DISCUSSION}

Factor B is activated by binding surface-bound C3b and subsequent cleavage by factor D. Our data reveal the conformation that keeps 
factor B locked in its proenzyme state. The structure adds this locked conformation to the open and closed conformations described in past studies of the VWA and I domains ${ }^{8,9}$ and provides insights into a previously uncharacterized mechanism for regulating serine protease activity $^{21}$. Assembly of an active protease complex probably proceeds through a number of steps. We hypothesize that initial binding of $\mathrm{C} 3 \mathrm{~b}$ dislocates the CCP triad. In turn, dislocation of the CCPs probably induces formation of an active MIDAS and allows access of the large C3b ligand. Concomitantly, the blocking helix $\alpha \mathrm{L}$ is displaced from its position in the VWA domain, thereby allowing the activation helix $\alpha 7$ to move into its normal location. Rearrangement of helices $\alpha \mathrm{L}$ and $\alpha 7$ liberates the bound scissile peptide, making it accessible for proteolytic cleavage by factor D. Cleavage of the scissile bond results in dissociation of the $\mathrm{Ba}$ fragment, yielding the active, short-lived protease complex that cleaves $\mathrm{C} 3$ into $\mathrm{C} 3 \mathrm{a}$ and $\mathrm{C} 3 \mathrm{~b}$. Thus, the tight regulation of complement activation is determined by a series of conformational changes that establish the $\mathrm{C} 3$ convertase activity required for amplification, which is crucial for the biological functions of the complement system.

\section{METHODS}

Protein expression, purification and crystallization. Human factor B fused to an N-terminal Gly-Ser-(His) ${ }_{6}$-Gly-Ser tag was expressed in human embryonic kidney $293 \mathrm{~S} \mathrm{GnTI}^{-}$cells to prevent complex and heterogeneous $\mathrm{N}$-linked glycosylation $^{25}$. Secreted factor B was purified from the expression medium via immobilized metal affinity chromatography followed by size-exclusion chromatography. The purified protein showed a single band of $90 \mathrm{kDa}$ on Coomassie-stained SDS-PAGE gels and on western blots probed with an antibody to $\mathrm{His}_{6}$. Details of factor B expression and purification are given in Supplementary Methods online. Crystals were grown by hanging drop vapor diffusion by mixing $1 \mu \mathrm{l}$ factor $\mathrm{B}\left(13 \mathrm{mg} \mathrm{m}^{-1}\right)$ in $10 \mathrm{mM}$ tris(hydroxymethyl)

\section{Table 1 Data collection and refinement statistics}

Factor B

\begin{tabular}{|c|c|}
\hline \multicolumn{2}{|l|}{ Data collection } \\
\hline Space group & $P 3_{1} 21$ \\
\hline \multicolumn{2}{|l|}{ Cell dimensions } \\
\hline$a, b, c(\AA)$ & $104.0,104.0,151.1$ \\
\hline$\alpha, \beta, \gamma\left(^{\circ}\right)$ & $90,90,120$ \\
\hline Resolution $(\AA)$ & $60.0-2.3(2.42-2.30$ \\
\hline$R_{\text {merge }}$ & $10.8(56.1)$ \\
\hline$I / \sigma I$ & $10.4(2.6)$ \\
\hline Completeness (\%) & $99.8(100)$ \\
\hline Redundancy & $4.1(4.1)$ \\
\hline \multicolumn{2}{|l|}{ Refinement } \\
\hline Resolution $(\AA)$ & $60.0-2.30$ \\
\hline No. reflections & 40,409 \\
\hline$R_{\text {work }} / R_{\text {free }}$ & $0.195 / 0.241$ \\
\hline \multicolumn{2}{|l|}{ No. atoms } \\
\hline Protein & 5,745 \\
\hline Ligand/ion & 78 \\
\hline Water & 295 \\
\hline \multicolumn{2}{|l|}{$B$-factors $\left(\AA^{2}\right)$} \\
\hline Protein & 33.5 \\
\hline Ligand/ion & 58.1 \\
\hline Water & 31.6 \\
\hline \multicolumn{2}{|l|}{ R.m.s. deviations } \\
\hline Bond lengths $(\AA)$ & 0.010 \\
\hline Bond angles $\left({ }^{\circ}\right)$ & 1.30 \\
\hline
\end{tabular}

Values in parentheses are for highest-resolution shell. aminomethane ( $\mathrm{pH} 7.4), 25 \mathrm{mM}$ arginine, $25 \mathrm{mM}$ glutamic acid and $1 \mu \mathrm{l}$ well solution $(50 \mathrm{mM}$ malic acid 2-(N-morpholino)ethanesulfonic acid tris (hydroxymethyl)aminomethane buffer (pH 6.5) and 12\% (w/v) PEG 1,500) at $20{ }^{\circ} \mathrm{C}$. Glycerol $(20 \% \mathrm{v} / \mathrm{v})$ was added to the well solution before flash-cooling of the crystal in liquid nitrogen. The crystal diffracted to $2.3-\AA$ resolution at the European Synchrotron Radiation Facility beamline ID14-EH4. The space group of the crystal was identified as $P 3_{1} 21(a=b=104.0$ and $c=151.1 \AA)$. Data were processed by MOSFLM and CCP4 (ref. 26). Crystallographic data collection and refinement statistics are given in Table 1.

Structure determination. The SP and VWA domain were placed by molecular replacement using Phaser ${ }^{27}$ with structures of the isolated SP (PDB 1DLE) $)^{28}$ and VWA (PDB 1Q0P) ${ }^{14}$ domains of factor B as search models. Subsequent placement of the three CCP domains using various homologous structures, as well as model completion by automated model building using ARP/wARP ${ }^{29}$, failed at this stage. The VWA helix $\alpha 7$ and SP domain loops C and D were rebuilt and the partial model was refined using COOT ${ }^{30}$, RESOLVE $^{31}$ and REFMAC5 (ref. 32). Using this partial model, ARP/wARP then successfully completed the model to $\sim 80 \%$. Iterative cycles of refinement with REFMAC5 and model building in COOT were used to finalize the model. The refined model of factor B contains 710 residues; residues 1-8, 218-232, 321-326 and 344-346 were excluded from the model because of poor electron density. $R$ and $R_{\text {free }}$ values were $19.5 \%$ and $24.1 \%$, respectively (see Table 1 for refinement statistics). All molecular graphic figures were generated with PyMOL (http:// pymol.sourceforge.net).

Accession codes. Protein Data Bank: Coordinates and structure factors have been deposited with accession code $2 \mathrm{OK} 5$.

Note: Supplementary information is available on the Nature Structural \& Molecular Biology website.

\section{ACKNOWLEDGMENTS}

We thank the European Synchrotron Radiation Facility for providing synchrotron radiation facilities and the beamline scientists at ID-14-EH4 for their help with data collection. This work was supported by a 'Pionier' program grant (P.G.) of the Council for Chemical Sciences of the Netherlands Organization for Scientific Research (NWO-CW).

\section{COMPETING INTERESTS STATEMENT}

The authors declare that they have no competing financial interests.

Published online at http://www.nature.com/nsmb/

Reprints and permissions information is available online at http://npg.nature.com/ reprintsandpermissions

1. Carroll, M.C. The complement system in regulation of adaptive immunity. Nat. Immunol. 5, 981-986 (2004).

2. Walport, M.J. Complement. First of two parts. N. Engl. J. Med. 344, 1058-1066 (2001).

3. Fishelson, Z., Pangburn, M.K. \& Muller-Eberhard, H.J. Characterization of the initial C3 convertase of the alternative pathway of human complement. J. Immunol. 132, 1430-1434 (1984).

4. Xu, Y., Narayana, S.V. \& Volanakis, J.E. Structural biology of the alternative pathway convertase. Immunol. Rev. 180, 123-135 (2001).

5. Pangburn, M.K. \& Muller-Eberhard, H.J. The C3 convertase of the alternative pathway of human complement. Enzymic properties of the bimolecular proteinase. Biochem. J. 235, 723-730 (1986)

6. Pryzdial, E.L. \& Isenman, D.E. Alternative complement pathway activation fragment Ba binds to C3b. Evidence that formation of the factor B-C3b complex involves two discrete points of contact. J. Biol. Chem. 262, 1519-1525 (1987).

7. Horiuchi, T., Macon, K.J., Engler, J.A. \& Volanakis, J.E. Site-directed mutagenesis of the region around Cys-241 of complement component C2. Evidence for a C4b binding site. J. Immunol. 147, 584-589 (1991).

8. Emsley, J., Knight, C.G., Farndale, R.W., Barnes, M.J. \& Liddington, R.C. Structural basis of collagen recognition by integrin alpha2beta1. Cell 101, 47-56 (2000).

9. Springer, T.A. Complement and the multifaceted functions of VWA and integrin I domains. Structure 14, 1611-1616 (2006).

10. Ponnuraj, K. et al. Structural analysis of engineered Bb fragment of complement factor $\mathrm{B}$ : insights into the activation mechanism of the alternative pathway C3-convertase. Mol. Cell 14, 17-28 (2004).

11. Milder, F.J. et al. Structure of complement component C2a: implications for convertase formation and substrate binding. Structure 14, 1587-1597 (2006). 
12. Smith, C.A., Vogel, C.W. \& Muller-Eberhard, H.J. MHC Class III products: an electron microscopic study of the C3 convertases of human complement. J. Exp. Med. 159, 324-329 (1984).

13. Shimaoka, M. et al. Structures of the alpha L I domain and its complex with ICAM-1 reveal a shape-shifting pathway for integrin regulation. Cell 112, 99-111 (2003).

14. Bhattacharya, A.A., Lupher, M.L., Jr., Staunton, D.E. \& Liddington, R.C. Crystal structure of the A domain from complement factor B reveals an integrin-like open conformation. Structure 12, 371-378 (2004).

15. Emsley, J., King, S.L., Bergelson, J.M. \& Liddington, R.C. Crystal structure of the I domain from integrin alpha2beta1. J. Biol. Chem. 272, 28512-28517 (1997).

16. Fishelson, Z., Pangburn, M.K. \& Muller-Eberhard, H.J. C3 convertase of the alternative complement pathway. Demonstration of an active, stable $\mathrm{C} 3 \mathrm{~b}, \mathrm{Bb}(\mathrm{Ni})$ complex. J. Biol. Chem. 258, 7411-7415 (1983).

17. Hourcade, D.E., Mitchell, L., Kuttner-Kondo, L.A., Atkinson, J.P. \& Medof, M.E. Decayaccelerating factor (DAF), complement receptor 1 (CR1), and factor $\mathrm{H}$ dissociate the complement AP C3 convertase (C3bBb) via sites on the type A domain of $\mathrm{Bb}$. J. Biol. Chem. 277, 1107-1112 (2002)

18. Hourcade, D.E., Mitchell, L.M. \& Oglesby, T.J. Mutations of the type A domain of complement factor B that promote high-affinity C3b-binding. J. Immunol. 162, 2906-2911 (1999).

19. Williams, S.C., Hinshelwood, J., Perkins, S.J. \& Sim, R.B. Production and functional activity of a recombinant von Willebrand factor-A domain from human complement factor B. Biochem. J. 342, 625-632 (1999).

20. Kam, C.M. et al. Human complement proteins D, C2, and B. Active site mapping with peptide thioester substrates. J. Biol. Chem. 262, 3444-3451 (1987).

21. Khan, A.R. \& James, M.N. Molecular mechanisms for the conversion of zymogens to active proteolytic enzymes. Protein Sci. 7, 815-836 (1998).

22. Hourcade, D.E., Wagner, L.M. \& Oglesby, T.J. Analysis of the short consensus repeats of human complement factor B by site-directed mutagenesis. J. Biol. Chem. 270, 19716-19722 (1995).
23. Xu, Y. \& Volanakis, J.E. Contribution of the complement control protein modules of $\mathrm{C} 2$ in C4b binding assessed by analysis of C2/factor B chimeras. J. Immunol. 158, 5958-5965 (1997).

24. Thurman, J.M. et al. A novel inhibitor of the alternative complement pathway prevents antiphospholipid antibody-induced pregnancy loss in mice. Mol. Immunol. 42, 87-97 (2005).

25. Reeves, P.J., Callewaert, N., Contreras, R. \& Khorana, H.G. Structure and function in rhodopsin: high-level expression of rhodopsin with restricted and homogeneous $\mathrm{N}$-glycosylation by a tetracycline-inducible $\mathrm{N}$-acetylglucosaminyltransferase I-negative HEK293S stable mammalian cell line. Proc. NatI. Acad. Sci. USA 99, 13419-13424 (2002).

26. Collaborative Computational Project, Number 4. The CCP4 suite: programs for protein crystallography. Acta Crystallogr. D Biol. Crystallogr. 50, 760-763 (1994).

27. Storoni, L.C., McCoy, A.J. \& Read, R.J. Likelihood-enhanced fast rotation functions Acta Crystallogr. D Biol. Crystallogr. 60, 432-438 (2004).

28. Jing, $\mathrm{H}$. et al. New structural motifs on the chymotrypsin fold and their potential roles in complement factor B. EMBO J. 19, 164-173 (2000).

29. Perrakis, A., Morris, R. \& Lamzin, V.S. Automated protein model building combined with iterative structure refinement. Nat. Struct. Biol. 6, 458-463 (1999).

30. Emsley, P. \& Cowtan, K. Coot: model-building tools for molecular graphics. Acta Crystallogr. D Biol. Crystallogr. 60, 2126-2132 (2004).

31. Terwilliger, T.C. Automated main-chain model building by template matching and iterative fragment extension. Acta Crystallogr. D Biol. Crystallogr. 59, 38-44 (2003).

32. Winn, M.D., Isupov, M.N. \& Murshudov, G.N. Use of TLS parameters to mode anisotropic displacements in macromolecular refinement. Acta Crystallogr. D Biol. Crystallogr. 57, 122-133 (2001).

33. Janssen, B.J., Christodoulidou, A., McCarthy, A., Lambris, J.D. \& Gros, P. Structure of $\mathrm{C} 3 \mathrm{~b}$ reveals conformational changes that underlie complement activity. Nature 444 213-216 (2006).

34. Wiesmann, C. et al. Structure of $\mathrm{C} 3 \mathrm{~b}$ in complex with CRIg gives insights into regulation of complement activation. Nature 444, 217-220 (2006). 